\title{
Classification of pancreatitis
}

\author{
M SARNER AND P B COTTON \\ International Workshop, King's College, Cambridge, 23-25 March 1983
}

An international group of doctors* interested in pancreatic disease met in Cambridge in March 1983, under the auspices of the Pancreatic Society of Great Britain and Ireland, to discuss the classification of pancreatitis in the light of developments that have taken place in the 20 years since the crucial conference in Marseille. ${ }^{1}$

The Marseille delegates had separated pancreatitis into four main groups: (1) acute, (2) relapsing acute (with clinical and biological restitution of the gland), (3) chronic relapsing (chronic pancreatitis with acute exacerbations). (4) chronic (with anatomical and/or functional residual damage to the gland).

This classification has had a profound influence on the study of pancreatitis but some problems have persisted. Clinicians can recognise acute first onset pancreatitis and define advanced chronic pancreatitis, but difficulties of definition have arisen between these extremes. The distinction between relapsing acute and chronic relapsing pancreatitis is usually made on grounds of function, as pathological specimens are rarely available - but most patients with pancreatic disease are managed without function tests. The fact that the official Marseille publication is not easily available has led to misquotation and some misunderstanding. One implication that has been taken is that alcohol abuse leads only to chronic pancreatitis; it is now appreciated that alcohol induced pancreatitis may present acutely and that alcohol induced disease is not inevitably progressive.

\footnotetext{
* Prof Dr M Cremer - Belgium. Dr H Worning - Denmark. Prof J-C Sarles - France. Prof I N Marks - South Africa. Prof I Ihse Sweden. Prof Dr R Amman - Switzerland. Prof G Adler - West Germany. Prof Dr M Classen - West Germany. Prof Dr W Creutzfeld - West Germany. Dr O A Gebhardt - West Germany. Dr P G Lankisch - West Germany. Prof Dr P Schmitz-Moormann West Germany. Prof L Carey - USA. Dr P C Freeny - USA. Prof C F Frev - USA. Prof J H Ranson - USA. Prof H A Reber - USA. and from the UK Dr A Axon. Dr W K Blenkinsop. Mr R E C Collins. Dr P B Cotton. Dr A K Foulis, Mr G Glazer. Prof H T Howat. Mr C W Imric. Dr W Lees, Mr M J McMahon. Dr C N Mallinson. Mr R C G Russell, Dr M Sarner. Mr B Torrance. Mr J E Trapnell. Prof R C N Williamson.

Received for publication 9 December 1983
}

Clinicians not familiar with the details of the Marseille classification have often used 'acute' and 'chronic' in a temporal sense, analogous to their usage in liver disease. Chronic hepatitis means a persisting problem - but does not necessarily imply irreversible damage. Chronic pancreatitis, strictly speaking, means irreversible change.

New pancreatic imaging methods have become popular during the last decade, and these, in conjunction with function data, provide some measure of organ damage. Disease activity is less easy to define, unless the tests are repeated. As yet, no subgroup markers are fully validated.

\section{Specialist views}

Many different scientific disciplines are interested in pancreatitis, and their specialists look at the disease from different points of view. The Workshop therefore split initially into five speciality groups, these being: demography and aetiology, exocrine function, imaging, histopathology, and surgery.

Each group reviewed progress within its particular field of interest and attempted to define types of pancreatitis which they could recognise.

\section{Aetiology}

Aetiology has crucial prognostic value, as pancreatitis may be halted once the cause is removed. Thus it is recognised that pancreatitis caused by gall stones almost never leads to chronic disease. The importance of alcohol has also been fully substantiated. There is no threshold of alcohol consumption above which pancreatitis seems invevitable; most alcoholics do not have clinically evident pancreatic disease and those factors which determine disease in an individual are yet to be 
defined. Sensitivity markers would have a central place in such an aetiological classification but are not yet available; further studies on proteins such as lactoferrin and stone protein or tissue characterisers such as HLA antigens are important. The cause of the types of chronic calcifying pancreatitis found in Africa and India remain obscure: more detailed nutritional studies are required. Drug induced acute pancreatitis has become better recognised and is perhaps more common than hitherto. The hypothesis that the congenital anomaly of pancreas divisum is able to cause obstructive pancreatitis has been proposed.

\section{Exocrine function}

Biochemical tests have an important role in the diagnosis and classification of pancreatitis. Serum amylase (or iso-amylase) and lipase remain the most reliable tests for acute pancreatitis; values greater than $10 \mathrm{SD}$ above the laboratory mean $(5 \times$ upper normal limit) are diagnostic. Levels may return to normal within 48-72 hours of the acute episode after which more information may be obtained by imaging. It is recognised that high concentrations of serum amylase may occur without acute inflammation - for example, in patients with 'cysts' - and that normal concentrations may be found in acute inflammation when the pancreas is chronically diseased. Impairment of exocrine function may persist for some long time after a single acute attack.

Pancreatic exocrine reserve is best measured by duodenal aspiration after direct stimulation of the gland, with assay of bicarbonate and enzyme protein output. Duodenal drainage tests detect pancreatic insufficiency when damage is marked or moderate, but do not predict progress of the disease or help to define aetiology. Markers in duodenal aspirate or pancreatic juice - for example, lactoferrin, stone protein, and trypsin inhibitor - may be more informative.

Indirect tests of pancreatic function - for example, pancreatic polypeptide release, radioimmunoassay of trypsin, pancreatic iso-amylase inhibitor, and tubeless tests such as the pancreolauryl test, can define severe pancreatic insufficiency, but are insensitive at earlier stages of the disease.

Sequential tests of pancreatic function are necessary to define whether the disease is recovering, static, or progressive.

\section{Imaging}

Endoscopic pancreatography (ERP) has been widely used in patients with known or suspected chronic pancreatitis; its role in patients with acute pancreatitis has yet to be defined. The imaging group emphasised the importance of high quality radiographs, and proposed new definitions and gradings of pancreatogram changes in pancreatitis: normal, equivocal (normal except for fewer than three abnormal side branches), mild (more than three abnormal side branches but normal main duct), moderate (abnormal main duct with or without branch changes), and marked (as moderate pancreatitis but also at least one of the following duct obstruction, severe irregularity or dilatation, ductal filling defects, or a cavity).

The sensitivity and specificity of ultrasound and computed tomography scanning are also dependent upon quality: observer variation has restricted the general acceptance of ultrasound scanning. Agreed criteria for grading are shown in the Table.

The group emphasised that imaging abnormalities did not always correlate with the state of the gland as judged by other methods (histopathology and function testing) and that pancreatograms and scans can be normal in patients with histologically proven disease.

\section{Histopathology}

The histopathology group distinguished only acute and chronic varieties of pancreatitis: some specimens contain features of both.

The initial phase of acute pancreatitis is conveniently called oedematous although this lesion is difficult to identify in human material. The earliest experimental changes (three to six hours after a defined insult) include separation of duct epithelial cells, with swelling and disruption of cytoplasmic organelles in the acinar cells. Later there is fat necrosis and/or necrosis of pancreatic tissue (some of which is because of vascular occlusion); either of these features may be dominant, and both can be associated with haemorrhage.

Two types of acute damage can be recognised: necrosis of duct epithelia with periductal acute inflammation - and perilobular necrosis probably owing to hypoperfusion. Chronic inflammatory changes include fibrosis, duct abnormalities, calcification, and inflammatory infiltrate. Fibrosis (particularly perilobular and panlobular) indicates a previous inflammatory process. Segmental duct dilatation and alternating dilatation and stenosis are more specific than general dilatation. Pancreatic islets are normally unaffected but may show compensatory hypertrophy.

Pathological definitions of pancreatitis are confused by the fact that similar changes are seen in elderly patients without known pancreatic disease. 
Table Chronic pancreatitis - Image grading

\begin{tabular}{|c|c|c|c|}
\hline & \multicolumn{2}{|l|}{$E R P$} & US and $C T$ \\
\hline 1 Normal & \multicolumn{3}{|c|}{ Quality study visualising whole gland without abnormal features } \\
\hline 2 Equivocal & $\begin{array}{l}\text { Less than three abnormal } \\
\text { branches }\end{array}$ & One sign only & $\left\{\begin{array}{l}\text { Main duct enlarged }(<4 \mathrm{~mm}) \\
\text { Gland enlarged (up to } 2 \times \mathrm{N}) \\
\text { Cavities }(<10 \mathrm{~mm})\end{array}\right.$ \\
\hline 3 Mild & $\begin{array}{l}\text { More than three abnormal } \\
\text { branches }\end{array}$ & Two or more signs & $\begin{array}{l}\text { Irregular ducts } \\
\text { Focal acute pancreatitis } \\
\text { Parenchymal heterogeneity }\end{array}$ \\
\hline 4 Moderate & $\begin{array}{l}\text { Abnormal main duct and } \\
\text { branches }\end{array}$ & & $\begin{array}{l}\text { Duct wall echoes increased } \\
\text { Irregular head/body contour }\end{array}$ \\
\hline 5 Marked & \multicolumn{3}{|c|}{$\begin{array}{l}\text { As above with one or more of: } \\
\qquad \begin{array}{l}\text { Large cavities }(>10 \mathrm{~mm}) \\
\text { Gross gland enlargement }(>2 \times \mathrm{N}) \\
\text { Intraduct filling defects or calculi } \\
\text { Duct obstruction, structure or gross irregularity } \\
\text { Contiguous organ invasion }\end{array}\end{array}$} \\
\hline
\end{tabular}

The ducts may be dilated and associated with fibrosis; microscopic foci of calcification, duct hyperplasia, metaplasia, and protein plugs are not uncommon.

\section{Surgery}

The surgeons were particularly concerned with defining severity in acute pancreatitis, in order to provide prognostic guidelines. The group separated mild acute pancreatitis (no multisystem failure with uncomplicated recovery), and severe acute pancreatitis (multisystem failure with early or late local or systemic complications), and defined certain local complications: phlegmon - an inflammatory mass in and around the pancreas; pseudocyst - a localised collection of fluid containing high concentrations of pancreatic enzymes within, adjacent or remote from the pancreas; abscess - pus in and around the pancreas.

Surgeons favour a classification which includes statements on aetiology, clinical status, functional, and morphological changes.

\section{Classification - consensus and problems}

Deliberations of the specialist groups led to broader discussion on classification. It was agreed to recommend retention of the terms 'acute pancreatitis' and 'chronic pancreatitis', despite the confusion which has arisen from their use.

\section{ACUTE PANCREATITIS}

This is defined as an acute condition typically presenting with abdominal pain, and usually associated with raised pancreatic enzymes in blood or urine, due to inflammatory disease of the pancreas.

\section{CHRONIC PANCREATITIS}

This is defined as a continuing inflammatory disease of the pancreas, characterised by irreversible morphological change, and typically causing pain and/or permanent loss of function.

Acute pancreatitis may recur. Many patients with chronic pancreatitis may have acute exacerbations but the condition may be completely painless.

Two main problems became apparent during the discussion. The first was to define acceptable criteria for 'irreversible morphological change' and 'loss of function'. Scans, pancreatograms and function studies are sensitive tests of severe disease, but less accurate in the early stages; comparative studies are difficult to interpret because techniques vary and an independent endpoint is often missing. The Workshop supported the idea of grading test results as proposed by the imaging group. Similar gradations should be defined for function tests. It would then be possible to agree criteria for diagnosis, which could depend upon a combination of tests (or a score) - for example, marked abnormalities in any one test, or moderate abnormalities in two, or mild changes in three (assuming a compatible clinical picture and exclusion of carcinoma).

The second main problem concerns the definition of patients who suffer repeated attacks of pancreatic pain, but in whom unequivocal morphological or functional abnormalities have not yet been shown. The Workshop discussed the need for a grouping 
intermediate between acute and chronic pancreatitis, perhaps only as a 'holding grade' before final classification. This concept was eventually rejected, it being assumed that most clinicians would naturally use the term 'probable chronic pancreatitis' where necessary.

\section{Conclusion}

The Marseille classification has been of great value in categorising pancreatic inflammatory disease, in particular the single acute event (acute pancreatitis) or the persisting disease state with residual damage (chronic pancreatitis). In practice the intermediate categories of 'relapsing acute' and 'chronic relapsing' are less easy to use. A single attack of acute pancreatitis may leave function and/or anatomy altered for some time. On the other hand, a patient with silent underlying disease may first present with an acute attack.

Only two clinical types of pancreatitis need be recognised - acute, or chronic with residual damage. Definitions should be expanded to include aetiology which predicts prognosis, and statements of function and morphological damage which can now be made more precisely. Markers in blood or pancreatic juice may eventually'define important subgroups. An agreed terminology is an essential basis for further understanding and study.

\section{Reference}

1 Sarles H. Pancreatitis. Symposium of Marseille 1963. Basel: Karger, 1965. 